\title{
Distribution-sensitivity of rank-dependent poverty measures
}

Citation for published version (APA):

Bosmans, K. G. M. (2012). Distribution-sensitivity of rank-dependent poverty measures. METEOR, Maastricht University School of Business and Economics. METEOR Research Memorandum No. 034 https://doi.org/10.26481/umamet.2012034

Document status and date:

Published: 01/01/2012

DOI:

10.26481/umamet.2012034

Document Version:

Publisher's PDF, also known as Version of record

\section{Please check the document version of this publication:}

- A submitted manuscript is the version of the article upon submission and before peer-review. There can be important differences between the submitted version and the official published version of record.

People interested in the research are advised to contact the author for the final version of the publication, or visit the DOI to the publisher's website.

- The final author version and the galley proof are versions of the publication after peer review.

- The final published version features the final layout of the paper including the volume, issue and page numbers.

Link to publication

\footnotetext{
General rights rights.

- You may freely distribute the URL identifying the publication in the public portal. please follow below link for the End User Agreement:

www.umlib.nl/taverne-license

Take down policy

If you believe that this document breaches copyright please contact us at:

repository@maastrichtuniversity.nl

providing details and we will investigate your claim.
}

Copyright and moral rights for the publications made accessible in the public portal are retained by the authors and/or other copyright owners and it is a condition of accessing publications that users recognise and abide by the legal requirements associated with these

- Users may download and print one copy of any publication from the public portal for the purpose of private study or research.

- You may not further distribute the material or use it for any profit-making activity or commercial gain

If the publication is distributed under the terms of Article $25 \mathrm{fa}$ of the Dutch Copyright Act, indicated by the "Taverne" license above, 


\section{Maastricht University}

Kristof Bosmans

Distribution-sensitivity of rankdependent poverty measures

$\mathrm{RM} / 12 / 034$

\section{METEOR}

Maastricht University School of Business and Economics

Maastricht Research School of Economics

of Technology and Organization

\section{PO. Box 616}

NL - 6200 MD Maastricht

The Netherlands 


\title{
Distribution-sensitivity of rank-dependent poverty measures*
}

\author{
Kristof Bosmans \\ Department of Economics, Maastricht University, \\ Tongersestraat 53, 6211 LM Maastricht, The Netherlands
}

April 4, 2012

\begin{abstract}
We propose a criterion to rank poverty measures on the basis of distribution-sensitivity. The criterion compares reactions to 'lossy' transfers among the poor and is sufficiently general to be applicable to any pair of poverty measures. We focus specifically on the class of rank-dependent poverty measures and provide distribution-sensitivity rankings of the poverty gap ratio, the first and second Sen measures, the Thon measure, the Shorrocks measure, and the Thon, Kakwani and S-Gini classes of measures. Moreover, we discuss the relationship between the proposed criterion and two alternative distribution-sensitivity criteria that directly apply the Arrow-Pratt theory of risk aversion. Finally, we provide an empirically tractable necessary and sufficient condition for unanimous poverty rankings by all continuous and replication invariant rank-dependent poverty measures exhibiting a predetermined minimum degree of distribution-sensitivity.
\end{abstract}

Keywords. Poverty · Distribution-sensitivity · Rank-dependent poverty measures $\cdot$ Inequality aversion $\cdot$ Risk aversion

JEL classification. D63

${ }^{*}$ I thank Koen Decancq, Lucio Esposito, Peter Lambert, Erwin Ooghe and Ernesto Savaglio for useful comments. All remaining shortcomings are my own.

E-mail: k.bosmans@maastrichtuniversity.nl 


\section{Introduction}

In two early contributions, Watts (1968) and Sen (1976) each stressed that poverty measures should be sensitive to the distribution of income among the poor. They argued that a given income increment matters more to a worse off poor individual than to a better off poor individual, and introduced some of the first modern poverty measures with the specific aim of incorporating this distributional aspect. The literature on poverty measurement that has developed since the pioneering contributions of Watts and Sen continues to recognize distribution-sensitivity as a major concern. 1

It is now common to assert that a proper poverty measure should satisfy at least a minimal degree of distribution-sensitivity, a requirement that usually comes in the form of a transfer property. But poverty measures typically go beyond this minimal degree of distribution-sensitivity and, moreover, differ considerably in the extent to which they go beyond. That is, some poverty measures generally tolerate greater sacrifices of mean income in return for a given distributional improvement than others. Although the literature has since the beginning regarded the degree of distribution-sensitivity as an important distinguishing factor between poverty measures, a formal definition of distribution-sensitivity comparisons has long been lacking $2^{2}$

Zheng (2000a) was the first to provide a solid theoretical foundation for comparisons of poverty measures on the basis of distribution-sensitivity. However, Zheng's distribution-sensitivity criterion, which is based on the Arrow-Pratt theory of risk aversion, applies only to the class of subgroupconsistent poverty measures. This can be considered as a severe limitation: Zheng's criterion does not allow distribution-sensitivity comparisons within the other major class of rank-dependent poverty measures, which includes important measures proposed by Sen (1976), Thon (1979, 1983), Kakwani (1980), Shorrocks (1995) and others. Zheng (2000a, p. 135) acknowledges this limitation and calls for an extension that would allow such comparisons.

The first objective of this paper is to formulate and discuss a new criterion of distribution-sensitivity that can compare also rank-dependent poverty measures. In line with Okun's (1975) 'leaky-bucket experiment', the proposed criterion compares reactions of poverty measures to 'lossy' transfers among the poor. Consider a transfer in which the better off individual foregoes an income amount $a+\ell$ while the worse off receives only an amount $a$.

\footnotetext{
${ }^{1}$ See Chakravarty (2009, Chapter 2), Lambert (2001, Chapter 6), Seidl (1988) and Zheng (1997) for surveys of this literature.

2The literature often uses the term 'poverty aversion' as a synonym for distributionsensitivity. However, this is somewhat of a misnomer because it is not the 'dislike toward poverty' that is the issue. See also Zheng (2000a, pp. 120-121).
} 
The larger the loss $\ell$ tolerated by a poverty measure - that is, the larger the loss $\ell$ can be without increasing poverty - the more distribution-sensitive the measure is considered to be. We demonstrate that the criterion translates into an easy-to-check condition on the weights of rank-dependent poverty measures and we use this result to compare the particular measures that have been proposed in the literature. It turns out that several of the established rank-dependent poverty measures can be conclusively ranked by the distribution-sensitivity criterion. For example, the Sen measure in general allows larger losses in transferring from better off to worse off than the Thon measure.

To gain further insight into the proposed distribution-sensitivity criterion, we compare it with two a priori plausible alternative criteria: a criterion based on lossy equalizations (creating complete equality among the poor at the cost of mean income) and Zheng's criterion. First, we show that the three criteria coincide on the class of subgroup-consistent poverty measures. Hence, the lossy transfer criterion and the lossy equalization criterion can be interpreted as two alternative generalizations of Zheng's criterion. Second, we demonstrate that the lossy equalization criterion is stronger than the lossy transfer criterion for the class of rank-dependent poverty measures. That is, the lossy equalization criterion ranks each pair of rank-dependent poverty measures that is ranked by the lossy transfer criterion, but in addition ranks pairs that are not ranked by the latter. We will argue, however, that at a conceptual level the lossy transfer criterion better captures the idea of distribution-sensitivity than the lossy equalization criterion.

The second objective of the paper is to explore unanimous rankings of income distributions by a class of poverty measures exhibiting a predetermined minimum degree of distribution-sensitivity. This application of the idea of distribution-sensitivity was suggested by Zheng (2000a), who was in turn inspired by Meyer's (1977) work on minimum risk averse unanimity rankings in the theory of choice under risk. Zheng examines the idea for the class of subgroup-consistent poverty measures. We present the complimentary analysis for the class of continuous and replication invariant rank-dependent poverty measures. An empirically tractable necessary and sufficient condition for two income distributions to be ranked by the minimum distributionsensitivity unanimity poverty ranking is provided. This unanimity ranking is moreover argued to be a useful extension of the standard concept of censored generalized Lorenz dominance.

The next section introduces notation and presents a brief overview of the rank-dependent poverty measures that have been proposed in the literature. Section 3 defines the main distribution-sensitivity criterion based on lossy transfers and applies it to obtain rankings of the established rank-dependent 
poverty measures. In Section 4, we consider the lossy equalization criterion and Zheng's criterion and discuss the relationships with the lossy transfer criterion. Finally, Section 5 discusses unanimity poverty rankings by measures exhibiting a predetermined minimum degree of distribution-sensitivity. All proofs are relegated to an appendix.

\section{Rank-dependent poverty measures}

The income of individual $i$ is a positive real number $x_{i}$ and the income distribution for a population of $n$ individuals is a vector $x=\left(x_{1}, x_{2}, \ldots, x_{n}\right)$ in $\mathbb{R}_{++}^{n}$. The set of income distributions for one or more individuals is $X=\bigcup_{n \in \mathbb{N}} \mathbb{R}_{++}^{n}$. For each income distribution $x$ in $X$, the incomes are ordered such that $x_{1} \leq x_{2} \leq \cdots \leq x_{n}$. The poverty line is an income level $z$ in $\mathbb{R}_{++}^{n}$. An individual $i$ is poor if $x_{i}<z$ and non-poor if $x_{i} \geq z$. For an income distribution $x$ in $X$, we write $n_{x}$ for the number of individuals and $q_{x}$ for the number of poor individuals. We drop the subscripts in $n_{x}$ and $q_{x}$ whenever this does not lead to confusion. For an income distribution $x$ in $X$, we write $\hat{x}$ for the censored income distribution $\left(x_{1}, x_{2}, \ldots, x_{q}, z, z, \ldots, z\right)$. A poverty measure is a function $P: X \rightarrow \mathbb{R}$. The value $P(x)$ is to be interpreted as the poverty level associated with income distribution $x$ in $X$.

A poverty measure $P$ is a rank-dependent poverty measure if, for each income distribution $x$ in $X$,

$$
P(x)=\sum_{i=1}^{q} w_{i}(q, n) \frac{z-x_{i}}{z},
$$

where $w_{1}(q, n) \geq w_{2}(q, n) \geq \cdots \geq w_{q}(q, n)>0.3$ All rank-dependent poverty measures satisfy the focus property (increasing the income of a non-poor individual does not affect poverty), monotonicity (decreasing the income of a poor individual increases poverty) and the transfer property (a transfer from a worse off poor individual to a better off poor individual, such that the latter does not cross the poverty line, does not decrease poverty). Note that the latter property already imposes a minimal degree of distribution-sensitivity.

Table 1 presents rank-dependent poverty measures that have been proposed in the literature (first two columns). The poverty gap ratio equates the weights of all income positions and hence disregards distribution. The two Sen (1976) measures were introduced with the explicit goal of bringing in distributional concerns. These two measures, as all subsequent measures

\footnotetext{
${ }^{3}$ The form in equation $(1)$ is relative. Multiplication by $z$ gives the absolute form. The results in this paper are not affected by the choice between these two forms.
} 
Table 1. Rank-dependent poverty measures

\begin{tabular}{ccc}
\hline Measure & $w_{i}(q, n)$ & $\frac{w_{i}(q, n)}{w_{j}(q, n)}$ \\
\hline Poverty gap ratio & $\frac{1}{n}$ & 1 \\
Sen & $\frac{2(q+1-i)}{(q+1) n}$ & $\frac{q+1-i}{q+1-j}$ \\
Second Sen & $\frac{2(q+0.5-i)}{q n}$ & $\frac{q+0.5-i}{q+0.5-j}$ \\
Thon & $\frac{2(n+1-i)}{(n+1) n}$ & $\frac{n+1-i}{n+1-j}$ \\
Shorrocks & $\frac{2(n+0.5-i)}{n^{2}}$ & $\frac{n+0.5-i}{n+0.5-j}$ \\
Kakwani class & $\frac{q(q+1-i)^{\kappa}}{n \sum_{i=1}^{q} i^{\kappa}}, \kappa \geq 0$ & $\left(\frac{q+1-i}{q+1-j}\right)^{\kappa}$ \\
Thon class & $\frac{\tau n+2-2 i}{(\tau-1) n^{2}}, \tau \geq 2$ & $\frac{\tau n+2-2 i}{\tau n+2-2 j}$ \\
S-Gini class & $\left(\frac{n+1-i}{n}\right)^{\sigma}-\left(\frac{n-i}{n}\right)^{\sigma}, \sigma \geq 1$ & $\frac{(n+1-i)^{\sigma}-(n-i)^{\sigma}}{(n+1-j)^{\sigma}-(n-j)^{\sigma}}$ \\
\hline
\end{tabular}

in the table, feature weights that strictly decrease with the income position. The Thon (1979) and Shorrocks (1995) measures satisfy a strong transfer property, which condemns regressive transfers even if the receiver crosses the poverty line, a property violated by the two Sen measures. The Kakwani (1980) class of poverty measures is based on the idea that transfers should have a greater impact on poverty if they take place further down in the income distribution. The Thon (1983) class, in contrast, reflects the idea that transfers should have the same impact irrespective of their location. For the Kakwani class, the poverty gap ratio is obtained for $\kappa=0$ and the Sen measure for $\kappa=1$. The class of S-Gini poverty measures results from the combination of the S-Gini social welfare measure (Donaldson and Weymark, 1980) and Chakravarty's (1983, p. 79) welfare-based poverty measure. The poverty gap ratio is obtained for $\sigma=1$ and the Shorrocks measure for $\sigma=2$ (Chakravarty, 1997). A class that further generalizes the S-Gini class is considered in Section 5.

\section{A distribution-sensitivity criterion}

To compare poverty measures on the basis of distribution-sensitivity, we compare their reactions to lossy transfers among the poor. Such a transfer increases the income of a worse off poor individual by a given amount, but decreases the income of a better off poor individual by a greater amount. 
Okun (1975, pp. 91-95) famously suggested the consideration of lossy transfers in order to gauge attitudes toward the tradeoff between the size and distribution of income 4 Let $x$ and $y$ be two income distributions in $X$. Then $x$ is said to be obtained from $y$ by a lossy transfer among the poor if $n_{x}=n_{y}=n, q_{x}=q_{y}=q$ and

$$
x=\left(y_{1}, y_{2}, \ldots, y_{i}+a, \ldots, y_{j}-b, \ldots, y_{q}, y_{q+1}, y_{q+2}, \ldots, y_{n}\right),
$$

where $0<a<b$ and $y_{i}<y_{i}+a \leq y_{j}-b<y_{j}$. A poverty measure $P$ is at least as distribution-sensitive as a poverty measure $R$ if $P$ registers a poverty reduction for each lossy transfer among the poor for which $R$ does. In other words, given a transfer that benefits a worse off poor individual at the cost of a better off poor individual, $P$ tolerates at least as great a loss as $R$ does.

Definition 1. Let $P$ and $R$ be two poverty measures. Then $P$ is at least as distribution-sensitive as $R$ if $R(x)<R(y)$ implies $P(x)<P(y)$ for all $x$ and $y$ in $X$ such that $x$ is obtained from $y$ by a lossy transfer among the poor.

We say that $P$ is more distribution-sensitive than $R$ if $P$ is at least as distribution-sensitive as $R$ and $R$ is not at least as distribution-sensitive as $P$. We say that $P$ is equally distribution-sensitive as $R$ if $P$ is at least as distribution-sensitive as $R$ and $R$ is at least as distribution-sensitive as $P$.

The criterion in Definition 1 is sufficiently general to be applied to any pair of poverty measures. We focus specifically on the rank-dependent poverty measures. The following proposition rephrases distribution-sensitivity judgments in terms of conditions on the weights of rank-dependent poverty measures.

Proposition 1. Let $P_{w}$ and $P_{v}$ be two rank-dependent poverty measures. Then the following two conditions are equivalent:

(i) $P_{w}$ is at least as distribution-sensitive as $P_{v}$,

(ii) for all $i, j, q$ and $n$ such that $1 \leq i<j \leq q \leq n$,

$$
\frac{w_{i}(q, n)}{w_{j}(q, n)} \geq \frac{v_{i}(q, n)}{v_{j}(q, n)} .
$$

Note that (ii) is equivalent to the condition that, for all $q$ and $n$ such that $q \leq n$, the weight vector $\left(w_{1}(q, n), w_{2}(q, n), \ldots, w_{q}(q, n)\right)$ dominates the weight vector $\left(v_{1}(q, n), v_{2}(q, n), \ldots, v_{q}(q, n)\right)$ in relative differentials (Moyes, 1994, p. 276, Marshall, Olkin and Arnold, 2011, p. 186).

\footnotetext{
${ }^{4}$ See also Atkinson (1973, pp. 66-67).
} 
We now compare the rank-dependent poverty measures defined in the previous section on the basis of distribution-sensitivity. Condition (ii) can easily be checked for the weight ratios presented in the final column of Table 1. Using $\succ$ to denote the relation 'is more distribution-sensitive than', the ranking of the first five measures reads as follows:

$$
\text { Second Sen } \succ \text { Sen } \succ \text { Thon } \succ \text { Poverty gap ratio } \bigsqcup^{5}
$$

and

$$
\text { Second Sen } \succ \text { Shorrocks } \succ \text { Thon. }
$$

The Sen and Shorrocks measures cannot in general be ranked on the basis of distribution-sensitivity. However, the Sen measure is more distributionsensitive than the Shorrocks measure on the restricted domain of income distributions with $q<n$, while the reverse ranking holds on the restricted domain of income distributions with $n=q$. The former domain restriction is obviously more empirically relevant than the latter. Next, the Thon measure is equally distribution-sensitive as the member of the Thon class for which $\tau=2$. Finally, distribution-sensitivity increases with $\kappa$ for the Kakwani class, decreases with $\tau$ for the Thon class and increases with $\sigma$ for the S-Gini class.

\section{Two alternative distribution-sensitivity criteria}

To put the distribution-sensitivity criterion proposed in the previous section into perspective, we contrast it with two plausible alternative criteria. These two criteria have in common that they are direct applications of the ArrowPratt theory of risk aversion, an established source of inspiration for the analysis of inequality aversion (Lambert, 2001, Chapter 4).

The first alternative criterion compares poverty measures by their reactions to lossy equalizations among the poor. Such an operation equalizes the incomes of all poor individuals, but diminishes the total income of the poor. Let $x$ and $y$ be two income distributions in $X$. Then $x$ is obtained from $y$ by a lossy equalization among the poor if $n_{x}=n_{y}=n, q_{x}=q_{y}=q$ and

$$
x=\left(c, c, \ldots, c, y_{q+1}, y_{q+2}, \ldots, y_{n}\right),
$$

where $c q<\sum_{i=1}^{q} x_{i}$. A poverty measure $P$ is at least as distribution-sensitive as a poverty measure $R$ if $P$ registers a poverty reduction for each lossy equalization among the poor for which $R$ does. Because the criterion measures

\footnotetext{
${ }^{5}$ The finding that the Sen measure is more distribution-sensitive than the Thon measure confirms an intuition expressed by Zheng (2000a, p. 135).
} 
the maximum income amount that can be sacrificed in exchange for complete equality, it is an immediate translation of the Arrow-Pratt criterion of risk aversion to the poverty context. ${ }^{6}$

Definition 2. Let $P$ and $R$ be two poverty measures. Then $P$ is at least as distribution-sensitive ${ }^{*}$ as $R$ if $R(x)<R(y)$ implies $P(x)<P(y)$ for all $x$ and $y$ in $X$ such that $x$ is obtained from $y$ by a lossy equalization among the poor.

The second alternative distribution-sensitivity criterion we consider was proposed by Zheng (2000a). Zheng focuses exclusively on the subgroupconsistent poverty measures. A poverty measure $P$ is a subgroup-consistent poverty measure if, for each income distribution $x$ in $X$,

$$
P(x)=\frac{1}{n} \sum_{i=1}^{q} p_{z}\left(x_{i}\right),
$$

where $p_{z}: \mathbb{R}_{++} \rightarrow \mathbb{R}$ is strictly decreasing on $(0, z]$, continuous and convex, with $p_{z}(t)=0$ for each $t \geq z$. In line with the Arrow-Pratt criterion of risk aversion, Zheng (2000a, Proposition 1) measures distribution-sensitivity by the 'degree of convexity' of the function $p_{z}$.

Definition 3. Let $P_{p}$ and $P_{r}$ be two subgroup-consistent poverty measures. Then $P_{p}$ is at least as distribution-sensitive** as $P_{r}$ if there is a convex function $\psi$ such that $p_{z}(t)=\psi\left(r_{z}(t)\right)$ for each $t$ in $(0, z]$.

We first consider how the three criteria in Definitions 1, 2 and 3 compare subgroup-consistent poverty measures. The following proposition shows that the three criteria coincide for this class. Hence, the lossy transfer criterion in Definition 1 and the lossy equalization criterion in Definition 2 can be seen as two alternative generalizations of Zheng's criterion in Definition 3.

\footnotetext{
${ }^{6}$ The criterion is similar to the formulation of the Arrow-Pratt criterion in Definition (v) of Mas-Colell, Whinston and Green (1995, p. 191). Incidentally, note that the criterion in Definition 2 can be rephrased in terms of the equally distributed equivalent income, the analogue of the certainty equivalent in the theory of choice under risk. For a poverty measure $P$ and an income distribution $x$ in $X$, the equally distributed equivalent income $e_{P}(x)$ is defined by $P\left(e_{P}(x), e_{P}(x), \ldots, e_{P}(x), x_{q+1}, x_{q+2}, \ldots, x_{n}\right)=P(x)$. The next result follows easily from Bosmans (2007a, Theorem 1 (a)): for two poverty measures $P$ and $R$ such that $e_{P}(x)$ and $e_{R}(x)$ exist for each $x$ in $X$, we have that $P$ is at least as distribution sensitive* $^{*}$ as $R$ if and only if $e_{P}(x) \leq e_{R}(x)$ for each $x$ in $X$.
} 
Proposition 2. Let $P_{p}$ and $P_{r}$ be two subgroup-consistent poverty measures. Then the following three conditions are equivalent:

(i') $P_{p}$ is at least as distribution-sensitive as $P_{r}$,

(ii') $P_{p}$ is at least as distribution-sensitive* as $P_{r}$,

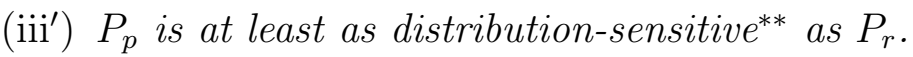

Next we consider distribution-sensitivity comparisons of rank-dependent poverty measures. The following proposition restates the criterion in Definition 2 in terms of the weights of rank-dependent poverty measures. For a rank-dependent poverty measure $P_{w}$, we write $\bar{w}_{i}(q, n)$ for the normalized weight $w_{i}(q, n) / \sum_{i=1}^{q} w_{i}(q, n)$.

Proposition 3. Let $P_{w}$ and $P_{v}$ be two rank-dependent poverty measures. Then the following two conditions are equivalent:

(i") $P_{w}$ is at least as distribution-sensitive* as $P_{v}$,

(ii") for all $q$ and $n$ such that $1 \leq q \leq n$, and for each $k=1,2, \ldots, q$,

$$
\sum_{i=1}^{k} \bar{w}_{i}(q, n) \geq \sum_{i=1}^{k} \bar{v}_{i}(q, n) .
$$

Condition (ii") is equivalent to the condition that, for all $q$ and $n$ such that $q \leq n$, the normalized weight vector $\left(\bar{w}_{1}(q, n), \bar{w}_{2}(q, n), \ldots, \bar{w}_{q}(q, n)\right)$ is majorized by the normalized weight vector $\left(\bar{v}_{1}(q, n), \bar{v}_{2}(q, n), \ldots, \bar{v}_{q}(q, n)\right)$. It follows from Marshall, Olkin and Arnold (2011, Proposition B.1, p. 186) that condition (ii) of Proposition 1 implies condition (ii"). $]^{7}$ The reverse implication does not hold. Hence, for the rank-dependent poverty measures, the lossy equalization criterion is stronger than the lossy transfer criterion. That is, each pair of rank-dependent poverty measures ranked by the lossy transfer criterion is ranked equivalently by the lossy equalization criterion, and there exist pairs of rank-dependent poverty measures that are ranked by the latter but not by the former.

We conclude this section by arguing that the lossy transfer criterion better captures the concept of distribution-sensitivity than the lossy equalization criterion.

In its minimal form, distribution-sensitivity requires that a small transfer from a better off poor individual to a worse off poor individual decreases poverty. We contrast the egalitarian and prioritarian justifications for this requirement. The egalitarian justification would be that inequality among

\footnotetext{
${ }^{7}$ See also Marshall, Olkin and Proschan (1967).
} 
the poor is in itself ethically undesirable and that the transfer decreases this inequality. The prioritarian justification does not rely on the intrinsic value of equality, but rather on the premise that additional units of income matter more the worse off an individual is (Parfit, 1995): because the transferred units go from a better to a worse off individual, the overall effect is a decrease of poverty. As argued extensively by Esposito and Lambert (2011), the poverty literature's interest in distribution-sensitivity seems to be driven more by a prioritarian attitude than by an egalitarian attitude.

The lossy transfer criterion in Definition 1 captures the prioritarian attitude well. By measuring the maximum allowed loss in transferring from a better off individual to a worse off individual, it directly measures the extent to which a poverty measure prioritizes the former over the latter ${ }^{8}$ The lossy equalization criterion in Definition 2, on the other hand, is clearly egalitarian in spirit 9 Moreover, as we have seen, for some pairs of poverty measures $P$ and $R$, the criterion concludes that $P$ is more distribution-sensitive than $R$, even though $R$ in some cases accepts a greater loss than $P$ in transferring from the better to the worse off, that is, even though $R$ in some cases gives more priority to the worse off than $P$. We conclude therefore that the lossy transfer criterion better expresses the prioritarian attitude that underlies the idea of distribution-sensitivity.

\section{Minimum distribution-sensitivity poverty rankings}

In empirical applications, it is interesting to identify rankings of income distributions agreed on by an appealing class of poverty measures ${ }^{10}$ Such rankings do not depend on the possibly arbitrary properties of any given single poverty measure and therefore allow drawing robust conclusions. We con-

\footnotetext{
${ }^{8}$ A restatement of Definition 1 may clarify this further. Let $x$ be obtained from $y$ by a lossy transfer from individual $j$ to individual $i$. Consider now a third income distribution $y^{\prime}=\left(y_{1}, y_{2}, \ldots, y_{i}, \ldots, y_{j}-b, \ldots, y_{q}, y_{q+1}, y_{q+2}, \ldots, y_{n}\right)$. Starting from $y^{\prime}$, the choice between $x$ and $y$ involves deciding which of the following two operations reduces poverty more: giving a smaller amount $a$ to the worse off individual $i$ (leading to $x$ ), or giving a greater amount $b$ to the better off individual $j$ (leading to $y$ ). This clearly gauges the extent to which the worse off gets priority over the better off. Note that the intuition that leads Zheng (2000a, pp. 119-120) to the Arrow-Pratt criterion is also prioritarian in spirit. Zheng considers the relative difference between, on the one hand, the poverty reduction caused by giving an income increment $d$ to the worse off poor individual and, on the other hand, the poverty reduction caused by giving the income increment $d$ to the better off individual.

${ }^{9}$ The lossy transfer criterion does not fare so well in egalitarian terms because the effect of a lossy transfer on inequality is ambiguous. See Seidl (2001) and Lambert and Lanza (2006).

${ }^{10}$ See Zheng (2000b) for a survey of the literature on unanimity poverty rankings.
} 
sider unanimous rankings by a class of rank-dependent poverty measures exhibiting a predetermined minimum degree of distribution-sensitivity. For the reason discussed at the end of the previous section, we focus solely on the lossy transfer distribution-sensitivity criterion in Definition 1. Our discussion complements Zheng's (2000a) analysis of minimum distribution-sensitivity rankings by the subgroup-consistent poverty measures.

We impose the properties continuity (small changes in incomes result in small changes in poverty) and replication invariance (replications of the income distribution leave poverty unchanged) ${ }^{11}$ The set $\mathcal{P}$ collects all rankdependent poverty measures that satisfy continuity and replication invariance. A poverty measure $P$ is in $\mathcal{P}$ if and only if $P$ is in the form of equation (1) and

$$
w_{i}(q, n)=f\left(\frac{n+1-i}{n}\right)-f\left(\frac{n-i}{n}\right),
$$

where $f:[0,1] \rightarrow \mathbb{R}$ is strictly increasing, continuous and convex. ${ }^{12}$ Because the weights do not depend on the number of poor individuals $q$, we will simplify by writing $w_{i}(n)$ instead of $w_{i}(q, n)$. Note that the class $\mathcal{P}$ contains the S-Gini measures (for $f: t \mapsto t^{\sigma}$ with $\sigma \geq 1$ ) and hence also the poverty gap ratio $(\sigma=1)$ and the Shorrocks measure $(\sigma=2)$.

Let $P_{w}$ in $\mathcal{P}$ be a predetermined poverty measure, to be interpreted as a desired lower bound on distribution-sensitivity. We are interested in unanimous rankings of income distributions by all poverty measures in $\mathcal{P}$ at least as distribution-sensitive as $P_{w}$. The following proposition provides a necessary and sufficient condition on two income distributions for such a unanimous ranking to hold. The two income distributions under comparison are assumed to have equal population sizes. Since any two income distributions can be replicated up to the same population size, this is without loss of generality. ${ }_{13}^{13}$

\footnotetext{
${ }^{11}$ The formal definitions of these properties are as follows. A poverty measure $P$ satisfies continuity if $P$ is continuous as a function of $x$ on $X$. A poverty measure $P$ satisfies replication invariance if, for all $x$ and $y$ in $X$ such that $n_{x}=k n_{y}$ and $x=(y, y, \ldots, y)$ with $k>1$, we have $P(x)=P(y)$. Note that the subgroup-consistent poverty measures, as defined in the previous section, satify these two properties as well.

${ }^{12}$ Continuity implies that the weights do not depend on the number of poor individuals. Given this fact and using replication invariance, equation (2) follows from Ebert (1988, Theorem 8).

${ }^{13}$ For a result in the spirit of Proposition 4 in the welfare context, see Capéau and Ooghe (2007, Proposition 3). The key difference with the result provided here is that they do not use a class of welfare measures analogous with $\mathcal{P}$, but rather the class of ' $r$-generalized Ginis', which do not satisfy replication invariance.
} 
Proposition 4. Let $P_{w}$ be a poverty measure in $\mathcal{P}$. Let $x$ and $y$ be two income distributions in $X$ such that $n_{x}=n_{y}=n$. Then the following two conditions are equivalent:

(a) $P(x) \leq P(y)$ for each poverty measure $P$ in $\mathcal{P}$ that is at least as distribution-sensitive as $P_{w}$,

(b) for each $k=1,2, \ldots, n$,

$$
\sum_{i=1}^{k} w_{i}(n)\left(z-\hat{x}_{i}\right) \leq \sum_{i=1}^{k} w_{i}(n)\left(z-\hat{y}_{i}\right) .
$$

Several remarks are in order. First, the unanimous ranking of two income distributions is preserved if the poverty line is substituted by a lower one. Clearly, if condition (b) is satisfied for some poverty line $z$, then it is also satisfied for all lower poverty lines. Hence, in empirical applications it suffices if the researcher is able to produce a reasonable upper bound for the poverty line. This is a convenient property in cases where the poverty line cannot be unambiguously determined (Atkinson, 1987).

Second, consider the following corollary of Proposition 4 obtained by choosing $P_{w}$ equal to the poverty gap ratio, that is, by choosing the lowest possible lower bound on distribution-sensitivity.

Corollary 1. Let $x$ and $y$ be two income distributions in $X$ such that $n_{x}=$ $n_{y}=n$. Then the following two conditions are equivalent:

(a') $P(x) \leq P(y)$ for each poverty measure $P$ in $\mathcal{P}$,

$\left(\mathrm{b}^{\prime}\right)$ for each $k=1,2, \ldots, n$,

$$
\sum_{i=1}^{k} z-\hat{x}_{i} \leq \sum_{i=1}^{k} z-\hat{y}_{i} .
$$

It was shown by Spencer and Fisher (1992), Jenkins and Lambert (1997, 1998) and Shorrocks (1998) that $\left(\mathrm{b}^{\prime}\right)$ is equivalent to the condition that $P(x) \leq P(y)$ for each subgroup-consistent poverty measure $P$. Condition $\left(\mathrm{b}^{\prime}\right)$ is moreover equivalent to the condition that $\hat{x}$ generalized Lorenz dominates $\hat{y}$, that is, $\sum_{i=1}^{k} \hat{x}_{i} \geq \sum_{i=1}^{k} \hat{y}_{i}$ for each $k=1,2, \ldots, n$. Hence, condition (b) of Proposition 4 can be regarded as a generalization of this concept of censored generalized Lorenz dominance ${ }^{14}$

\footnotetext{
${ }^{14}$ Zheng's (2000a, Proposition 3) analysis of minimum distribution-sensitivity rankings by the subgroup-consistent poverty measures yields an alternative generalization of censored generalized Lorenz dominance.
} 
Third, the question arises under what conditions on two income distributions $x$ and $y$ in $X$ there exist a $P_{w}$ in $\mathcal{P}$ such that condition (a) of Proposition 4 is satisfied. The next proposition provides an answer. Define the leximin relation $>_{\text {lex }}$ on $X$ as follows: for all income distributions $x$ and $y$ in $X$, we have $x>_{\text {lex }} y$ if and only if there is a $k \leq n$ such that $x_{i}=y_{i}$ for each $i<k$ and $x_{k}>y_{k}$.

Proposition 5. Let $x$ and $y$ be two income distributions in $X$ such that $\hat{x} \neq \hat{y}$ and $n_{x}=n_{y}=n$. Then the following two conditions are equivalent:

$\left(\mathrm{a}^{\prime \prime}\right)$ there is a poverty measure $P_{w}$ in $\mathcal{P}$ such that $P(x) \leq P(y)$ for each poverty measure $P$ in $\mathcal{P}$ that is at least as distribution-sensitive as $P_{w}$, $\left(\mathrm{b}^{\prime \prime}\right) \hat{x}>_{\text {lex }} \hat{y}$.

For any two income distributions $x$ and $y$ in $X$, exactly one of the following three is true: $\hat{x}=\hat{y}, \hat{x}>_{\operatorname{lex}} \hat{y}$ or $\hat{y}>_{\operatorname{lex}} \hat{x}$. This implies that there necessarily exists a lower bound on distribution-sensitivity such that $x$ and $y$ are unanimously ranked by all members of $\mathcal{P}$ respecting this lower bound. Clearly, if $\hat{x}=\hat{y}$, then all poverty measures in $\mathcal{P}$ agree that $x$ and $y$ are equally poor. The cases $\hat{x}>_{\operatorname{lex}} \hat{y}$ and $\hat{y}>_{\operatorname{lex}} \hat{x}$ are covered by Proposition $5{ }^{15}$

To conclude, we suggest an empirical approach in which the minimum distribution-sensitive poverty measure $P_{w}$ is determined by the data, instead of by an a priori choice. The idea is to determine the largest class of poverty measures that unanimously supports a given poverty judgment. For concreteness, let $P_{w}$ be a member of the class of S-Gini poverty measures. For a given pair of income distributions $x$ and $y$ in $X$, we then look for the least distribution-sensitive S-Gini measure $P_{w}$-that is, we look for the minimum value of $\sigma$-for which condition (b) in Proposition 4 is satisfied. The resulting value of $\sigma$ can be interpreted as an indicator of the strength of the poverty judgment: the lower this minimum value of $\sigma$, the larger (in terms of set inclusion) the class of minimum distribution-sensitive poverty measures agreeing on the poverty judgment.

Table 2 provides an illustration with five censored income distributions ${ }^{16}$ Consider, say, the comparison of $x$ and $s$. The value of 3.8 indicates that we have $P(s) \leq P(x)$ for each poverty measure $P$ in $\mathcal{P}$ at least as distributionsensitive as the S-Gini measure with $\sigma=3.8$. In addition, further lowering

\footnotetext{
${ }^{15}$ Proposition 5 also shows that the leximin relation defined on censored income distributions can be interpreted as a maximum distribution-sensitive poverty criterion. For closely related results in the welfare context, see Hammond (1975), Lambert (2001, Theorem 4.4) and Bosmans (2007b).

${ }^{16}$ Note that $x>_{\text {lex }} y>_{\text {lex }} s>_{\text {lex }} t>_{\text {lex }} u$. Hence, Proposition 5 implies that Table 2 is empty below the diagonal.
} 
Table 2. Minimum value of $\sigma$ such that the column income distribution is unanimously judged at least as poor as the row income distribution

\begin{tabular}{l|ccccc}
\hline & $x$ & $y$ & $s$ & $t$ & $u$ \\
\hline$x=(135,170,250, z, z)$ & & 1.3 & 3.8 & 3.0 & 4.3 \\
$y=(130,130,300, z, z)$ & - & & 7.6 & 4.5 & 4.9 \\
$s=(120,180,300, z, z)$ & - & - & & 1.0 & 3.8 \\
$t=(100,200,300, z, z)$ & - & - & - & & 6.0 \\
$u=(100,180, z, z, z)$ & - & - & - & - & \\
\hline
\end{tabular}

$\sigma$ results in loss of unanimity. Now consider the comparison of $s$ and $t$. The value of 1.0 indicates that $P(t) \leq P(s)$ for each poverty measure $P$ in $\mathcal{P}$ or, equivalently, that $t$ generalized Lorenz dominates $s$ (see Corollary 1 ). For these five income distributions, this would be the only conclusion that can be drawn using censored generalized Lorenz dominance. This illustrates the added value of the suggested approach: in addition to detecting cases of censored generalized Lorenz dominance $(\sigma=1.0)$, the approach allows to conclude, for example, that the poverty judgment for $x$ and $y(\sigma=1.3)$ comes relatively closer in strength to a censored generalized Lorenz dominance judgment than the poverty judgment for $t$ and $u(\sigma=6.0)$.

\section{References}

Atkinson AB, 1973. Non-mathematical summary. In: Atkinson AB (ed.) Wealth, Income and Inequality, pp. 64-71. Middlesex: Penguin.

Atkinson AB, 1987. On the measurement of poverty. Econometrica 55, 244-263.

Bosmans K, 2007a. Comparing degrees of inequality aversion. Social Choice and Welfare 29, 405-428.

Bosmans K, 2007b. Extreme inequality aversion without separability. Economic Theory 32, 589-594.

Capéau B, Ooghe E, 2007. On comparing heterogeneous populations: is there really a conflict between welfarism and a concern for greater equality in living standards? Mathematical Social Sciences 53, 128.

Chakravarty SR, 1983. Ethically flexible measures of poverty. Canadian Journal of Economics 16, 74-85.

Chakravarty SR, 1997. On Shorrock's reinvestigation of the Sen poverty index. Econometrica 65, 1241-1242.

Chakravarty SR, 2009. Inequality, Polarization and Poverty. Heidelberg: Springer.

Donaldson D, Weymark JA, 1980. A single-parameter generalization of the Gini indices of inequality. Journal of Economic Theory 22, 67-86.

Ebert U, 1988. Measurement of inequality: an attempt at unification and generalization. Social Choice and Welfare 5, 147-169. 
Esposito L, Lambert PJ, 2011. Poverty measurement: prioritarianism, sufficiency and the 'I's of poverty. Economics and Philosophy 27, 109-121.

Hammond PJ, 1975. A note on extreme inequality aversion. Journal of Economic Theory 11, 465-467.

Jenkins SP, Lambert PJ, 1997. Three 'I's of poverty curves, with an analysis of UK poverty trends. Oxford Economic Papers 49, 317-327.

Jenkins SP, Lambert PJ, 1998. Ranking poverty gap distributions: further TIPs for poverty analysis. In: Slottje DJ (ed.) Research on Economic Inequality, vol. 8, 31-38. Greenwich: JAI Press.

Kakwani N, 1980. On a class of poverty measures. Econometrica 48, 437-446.

Lambert PJ, 2001. The Distribution and Redistribution of Income, third edition. Manchester: Manchester University Press.

Lambert PJ, Lanza G, 2006. The effect on inequality of changing one or two incomes. Journal of Economic Inequality 4, 253-277.

Marshall AW, Olkin I, Arnold BC, 2011. Inequalities: Theory of Majorization and Its Applications, second edition. Heidelberg: Springer.

Marshall AW, Olkin I, Proschan F, 1967. Monotonicity of ratios of means and other applications of majorization. In: Shisha O (ed.) Inequalities, pp. 177-190. New York: Academic Press.

Mas-Colell A, Whinston MD, Green JR, 1995. Microeconomic Theory. Oxford: Oxford University Press.

Meyer J, 1977. Second degree stochastic dominance with respect to a function. International Economic Review 18, 477-487.

Moyes P, 1994. Inequality reducing and inequality preserving transformations of incomes: symmetric and individualistic transformations. Journal of Economic Theory 63, 271-298.

Okun AM, 1975. Equality and Efficiency: The Big Tradeoff. Washington, DC: Brookings Institution Press.

Parfit D, 1995. Equality or Priority? Lindley Lecture, Kansas University.

Seidl C, 1988. Poverty measurement: a survey. In: Böss D, Rose M, Seidl C (eds.) Welfare and Efficiency in Public Economics, 71-147. Heidelberg: Springer.

Seidl C, 2001. Inequality measurement and the leaky-bucket paradox. Economics Bulletin 4, 1-7.

Sen AK, 1976. Poverty: an ordinal approach to measurement. Econometrica 44, 219-231.

Shorrocks AF, 1995. Revisiting the Sen poverty index. Econometrica 63, 1225-1230.

Shorrocks AF, 1998. Deprivation profiles and deprivation indices. In: Jenkins SP, Kapteyn A, van Praag BMS (eds.) The Distribution of Welfare and Household Production: International Perspectives, 250-267. London: Cambridge University Press.

Spencer B, Fisher S, 1992. On comparing distributions of poverty gaps. Sankhyā: The Indian Journal of Statistics Series B 54, 114-126.

Thon D, 1979. On measuring poverty. Review of Income and Wealth 25, 429-440.

Thon D, 1983. A poverty measure. Indian Economic Journal 30, 55-70.

Watts HW, 1968. An economic definition of poverty. In: Moynihan DP (ed.) On Understanding Poverty, pp. 316-329. New York: Basic Books. 
Zheng B, 1997. Aggregate poverty measures. Journal of Economic Surveys 11, 123-162.

Zheng B, 2000a. Minimum distribution-sensitivity, poverty aversion, and poverty orderings. Journal of Economic Theory 95, 116-137.

Zheng B, 2000b. Poverty orderings. Journal of Economic Surveys 14, 427-466. 


\section{Appendix. Proofs}

Proof of Proposition 1. (i) $\Rightarrow$ (ii). Let $P_{w}$ and $P_{v}$ be two rank-dependent poverty measures. Assume that (i) holds. Seeking a contradiction, assume that (ii) does not hold. Let $i, j, q$ and $n$ be such that $1 \leq i<j \leq q \leq n$ and $w_{i}(q, n) / w_{j}(q, n)<v_{i}(q, n) / v_{j}(q, n)$. Let $y$ be an income distributions in $X$ such that $q_{y}=q, n_{y}=n$ and $y_{i}<y_{i+1} \leq y_{j-1}<y_{j}<z$. Let

$$
x=\left(y_{1}, y_{2}, \ldots, y_{i}+a, \ldots, y_{j}-b, \ldots, y_{q}, y_{q+1}, y_{q+2}, \ldots, y_{n}\right),
$$

where $0<a<b, y_{i}<y_{i}+a \leq y_{i+1} \leq y_{j-1} \leq y_{j}-b<y_{j}$ and $b / a=$ $v_{i}(q, n) / v_{j}(q, n)$. Note that $x$ is obtained from $y$ by a lossy transfer among the poor. Since $v_{i}(q, n) a-v_{j}(q, n) b=0$, we have $P_{v}(x)=P_{v}(y)$. And since $w_{i}(q, n) a-w_{j}(q, n) b<0$, we have $P_{w}(x)>P_{w}(y)$. This contradicts (i).

(ii) $\Rightarrow$ (i). Let $P_{w}$ and $P_{v}$ be two rank-dependent poverty measures. Assume that (ii) holds. Let $x$ and $y$ be two income distributions in $X$ such that $x$ is obtained from $y$ by a lossy transfer among the poor, i.e.,

$$
x=\left(y_{1}, y_{2}, \ldots, y_{i}+a, \ldots, y_{j}-b, \ldots, y_{q}, y_{q+1}, y_{q+2}, \ldots, y_{n}\right),
$$

where $0<a<b$ and $y_{i}<y_{i}+a \leq y_{j}-b<y_{j}$. Assume the transfer changes the position of $i$ to $i+k$ and the position of $j$ to $j-\ell$, i.e., $y_{i}+a=x_{i+k}$ and $y_{j}-b=x_{j-\ell}$. We decompose the amount $a$ into $k$ terms, one term for each position change. That is, $a=a_{0}+a_{1}+\cdots+a_{k}$ with $a_{t}=y_{i+t}-y_{i+t-1}$ for each $t=0,1, \ldots, k-1$ and $a_{k}=x_{i+k}-y_{i+k-1}$. We decompose the amount $b$ into $\ell$ terms, one term for each position change. That is, $b=b_{0}+b_{1}+\cdots+b_{\ell}$ with $b_{t}=y_{j-t+1}-y_{j-t}$ for each $t=0,1, \ldots, \ell-1$ and $b_{\ell}=y_{j-\ell+1}-x_{j-\ell}$. We have $P_{v}(x)-P_{v}(y)<0$ if and only if

$$
\begin{aligned}
& v_{i}(q, n) a_{0}+v_{i+1}(q, n) a_{1}+\cdots+v_{i+k}(q, n) a_{k} \\
& \quad-\left(v_{j-\ell}(q, n) b_{\ell}+v_{i+1}(q, n) b_{\ell-1}+\cdots+v_{j}(q, n) b_{0}\right)>0 .
\end{aligned}
$$

Since (ii) holds, we have

$$
\begin{aligned}
& \left(w_{i}(q, n), w_{i+1}(q, n), \ldots, w_{j}(q, n)\right) \\
& \quad=\left(\beta_{i} v_{i}(q, n), \beta_{i+1} v_{i+1}(q, n), \ldots, \beta_{j} v_{j}(q, n)\right),
\end{aligned}
$$

where $\beta_{i} \geq \beta_{i+1} \geq \cdots \geq \beta_{j} \geq 0$. Moreover, $a_{t} \geq 0$ for each $t=0,1, \ldots, k$ and $b_{t} \geq 0$ for each $t=0,1, \ldots, \ell$. Therefore, $P_{v}(x)-P_{v}(y)<0$ implies $P_{w}(x)-P_{w}(y)<0$.

Proof of Proposition 2. $\left(\mathrm{i}^{\prime}\right) \Rightarrow\left(\right.$ iii $\left.^{\prime}\right)$. Let $P_{p}$ and $P_{r}$ be two subgroupconsistent poverty measures. Assume that $\left(i^{\prime}\right)$ holds. Seeking a contradiction, 
assume that (iii') does not hold. Because $p_{z}$ and $r_{z}$ are continuous and strictly decreasing, there is a continuous and strictly increasing function $\psi$ such that $p_{z}(t)=\psi\left(r_{z}(t)\right)$ for each $t$ in $(0, z]$. Since (iii') does not hold, there is an interval $\left[r_{z}(t), r_{z}(s)\right]$, with $s<t<z$, on which $\psi$ is strictly concave. Note that this implies that $r_{z}$ is strictly convex on $[s, t]$. Let $a$ and $b$ be such that $0<a<b, s<s+a \leq t-b<t$ and

$$
r_{z}(s)-r_{z}(s+a)=r_{z}(t-b)-r(t) .
$$

Such $a$ and $b$ exist because $r_{z}$ is strictly convex on $[s, t]$. Since $\psi$ is strictly concave on $\left[r_{z}(t), r_{z}(s)\right]$ and $r_{z}(s)>r_{z}(s+a) \geq r_{z}(t-b)>r_{z}(t)$, we have

$$
p_{z}(s)-p_{z}(s+a)<p_{z}(t-b)-p_{z}(t) .
$$

Let $x$ and $y$ be two income distributions in $X$ such that

$$
y=\left(y_{1}, y_{2}, \ldots, t, \ldots, s, \ldots, y_{q}, y_{q+1}, y_{q+2}, \ldots, y_{n}\right)
$$

and

$$
x=\left(y_{1}, y_{2}, \ldots, t+a, \ldots, s-b, \ldots, y_{q}, y_{q+1}, y_{q+2}, \ldots, y_{n}\right) .
$$

Then $x$ is obtained from $y$ by a lossy transfer among the poor. Using equations (3) and (4), we obtain $P_{p}(x)>P_{p}(y)$ and $P_{r}(x)=P_{r}(y)$. This contra$\operatorname{dicts}\left(\mathrm{i}^{\prime}\right)$.

$\left(\right.$ iii $\left.^{\prime}\right) \Rightarrow\left(\mathrm{i}^{\prime}\right)$. Let $P_{p}$ and $P_{r}$ be two subgroup-consistent poverty measures. Let $x$ and $y$ be two income distributions in $X$ such that $x$ is obtained from $y$ by a lossy transfer among the poor, i.e.,

$$
x=\left(y_{1}, y_{2}, \ldots, y_{i}+a, \ldots, y_{j}-b, \ldots, y_{q}, y_{q+1}, y_{q+2}, \ldots, y_{n}\right),
$$

where $0<a<b$ and $y_{i}<y_{i}+a \leq y_{j}-b<y_{j}$. Let $P_{r}(x)<P_{r}(y)$. This implies

$$
r_{z}\left(y_{i}\right)-r_{z}\left(y_{i}+a\right)>r_{z}\left(y_{j}-b\right)-r_{z}\left(y_{i}\right) .
$$

If (iii') holds, then there is a strictly increasing and convex function $\psi$ such that $p_{z}(t)=\psi\left(r_{z}(t)\right)$ for each $t$ in $(0, z]$. Since $r_{z}\left(y_{i}\right)>r_{z}\left(y_{i}+a\right) \geq r_{z}\left(y_{j}-\right.$ b) $>r\left(y_{j}\right)$, we have

$$
\psi\left(r_{z}\left(y_{i}\right)\right)-\psi\left(r_{z}\left(y_{i}+a\right)\right)>\psi\left(r_{z}\left(y_{j}-b\right)\right)-\psi\left(r_{z}\left(y_{j}\right)\right) .
$$

Hence, $P_{p}(x)<P_{p}(y)$.

$\left(\right.$ ii' $\left.^{\prime}\right) \Leftrightarrow\left(\right.$ iii $\left.^{\prime}\right)$. This follows easily from Mas-Colell, Whinston and Green (1995, Proposition 6.C.2, p. 191). 
Proof of Proposition 3. For a rank-dependent poverty measure $P_{w}$ and an $x$ in $X$, the equally distributed equivalent income is $e_{w}(x)=\sum_{i=1}^{q} \bar{w}_{i}(q, n) x_{i}$. Note that, for all $x$ and $y$ in $X$ such that $n_{x}=n_{y}$ and $q_{x}=q_{y}$, we have $P_{w}(x) \leq P_{w}(y)$ if and only if $e_{w}(x) \geq e_{w}(y)$.

$\left(\mathrm{i}^{\prime \prime}\right) \Rightarrow\left(\mathrm{ii}^{\prime \prime}\right)$. Let $P_{w}$ and $P_{v}$ be two rank-dependent poverty measures. Assume that $\left(\mathrm{i}^{\prime \prime}\right)$ holds. Seeking a contradiction, assume that (ii") does not hold. Let $k, q$ and $n$ be such that $1 \leq k<q \leq n$ and

$$
\sum_{i=1}^{k} \bar{w}_{i}(q, n)<\sum_{i=1}^{k} \bar{v}_{i}(q, n) .
$$

Let $x$ be an income distribution in $X$ such that $n_{x}=n$ and $q_{x}=q$. Moreover, let $x_{i}=\alpha$ for each $i=1,2, \ldots, k$ and $x_{i}=\beta$ for each $i=k+1, k+2, \ldots, q$, with $\alpha<\beta$. Let $y$ be an income distribution in $X$ such that

$$
y=\left(c, c, \ldots, c, x_{q}, x_{q+1}, \ldots, x_{n}\right),
$$

where

$$
c=\alpha \sum_{i=1}^{k} \bar{w}_{i}(q, n)+\beta \sum_{i=k+1}^{q} \bar{w}_{i}(q, n)-\gamma
$$

with $\gamma>0$. Then $y$ is obtained from $x$ by a lossy equalization among the poor. We have $e_{w}(x)=c+\gamma$ and $e_{w}(y)=c$. Hence, $P_{w}(x)<P_{w}(y)$. Using equation (5), we obtain $e_{v}(x)<c+\gamma$. We also have $e_{v}(y)=c$. Hence, for a sufficiently small $\gamma>0$, we have $e_{v}(x)<e_{v}(y)$. Then $P_{v}(x)>P_{v}(y)$. This contradicts $\left(\mathrm{i}^{\prime \prime}\right)$.

$\left(\right.$ ii $\left.^{\prime \prime}\right) \Rightarrow\left(\mathrm{i}^{\prime \prime}\right)$. Let $P_{w}$ and $P_{v}$ be two rank-dependent poverty measures. Assume that (ii') holds. Then we have $e_{w}(x) \geq e_{v}(x)$ for each $x$ in $X$. Let $x$ and $y$ be two income distributions in $X$ such that $x$ is obtained from $y$ by a lossy equalization among the poor. Then we have $e_{w}(x)=e_{v}(x)$ and $e_{w}(y) \leq e_{v}(y)$. Hence, $P_{v}(x)<P_{v}(y)$ implies $P_{w}(x)<P_{w}(y)$.

Proof of Proposition 4. (a) $\Rightarrow(\mathrm{b})$. Let $P_{w}$ be a poverty measure in $\mathcal{P}$. Let $x$ and $y$ be two income distributions in $X$ such that $n_{x}=n_{y}=n$. Assume that (a) holds. Seeking a contradiction, assume that (b) does not hold. Let $k$ be such that

$$
\sum_{i=1}^{k} w_{i}(n)\left(z-\hat{x}_{i}\right)>\sum_{i=1}^{k} w_{i}(n)\left(z-\hat{y}_{i}\right) .
$$

Since $P_{w}$ is in $\mathcal{P}$, we have $w_{i}(m)=f((m+1-i) / m)-f((m-i) / m)$, where $f$ is strictly increasing, continuous and convex. Define $P_{v}$ as $v_{i}(m)=$ 
$g((m+1-i) / m)-g((m-i) / m)$ with

$$
g(t)= \begin{cases}\alpha f(t) & \text { for each } t \leq \frac{n-k}{n}, \\ f(t)-\beta & \text { for each } t \geq \frac{n-k}{n},\end{cases}
$$

where $0<\alpha<1$ and $\beta=(1-\alpha) f((n-k) / n)$. The function $g$ is strictly increasing, continuous and convex. Hence, $P_{v}$ is in $\mathcal{P}$.

We will now show that $P_{v}$ is more distribution-sensitive than $P_{w}$, i.e., for all $i, j$ and $m$ such that $1 \leq i<j \leq m$,

$$
\frac{v_{i}(m)}{v_{j}(m)} \geq \frac{w_{i}(m)}{w_{j}(m)}
$$

Note that

$$
\frac{m-j}{m}<\frac{m+1-j}{m} \leq \frac{m-i}{m}<\frac{m+1-i}{m}
$$

which means that there are five possible cases for the position of $(n-k) / n$.

- Case 1, $\frac{n-k}{\eta} \leq \frac{m-j}{m}$ : Then $v_{i}(m)=w_{i}(m)$ and $v_{j}(m)=w_{j}(m)$. Hence, equation (7) holds with equality.

- Case 2, $\frac{m-j}{m}<\frac{n-k}{n} \leq \frac{m+1-j}{m}$ : Then $v_{i}(m)=w_{i}(m)$. In addition,

$$
v_{j}(m)=f\left(\frac{m+1-j}{m}\right)-\beta-\alpha f\left(\frac{m-j}{m}\right)<w_{j}(m)
$$

because $\beta>(1-\alpha) f((m-j) / m)$. Hence, equation (7) holds strictly.

- Case 3, $\frac{m+1-j}{m}<\frac{n-k}{n} \leq \frac{m-i}{m}$ : Then $v_{i}(m)=w_{i}(m)$ and $v_{j}(m)=$ $\alpha w_{j}(m)<w_{j}(m)$. Hence, equation (7) holds strictly.

- Case $4, \frac{m-i}{m}<\frac{n-k}{n}<\frac{m+1-i}{m}$ : Then $v_{j}(m)=\alpha w_{j}(m)<w_{j}(m)$. In addition,

$$
v_{i}(m)=f\left(\frac{m+1-i}{m}\right)-\beta-\alpha f\left(\frac{m-i}{m}\right)>w_{i}(m)
$$

because $\beta<(1-\alpha) f((m+1-i) / m)$. Hence, equation (7) holds strictly.

- Case 5, $\frac{m+1-i}{m} \leq \frac{n-k}{n}$ : Then $v_{i}(m)=\alpha w_{i}(m)$ and $v_{j}(m)=\alpha w_{j}(m)$. Hence, equation (7) holds with equality. 
We have established that $P_{v}$ is in $\mathcal{P}$ and that $P_{v}$ is more distributionsensitive than $P_{w}$. Hence, (a) implies $P_{v}(x) \leq P_{v}(y)$, i.e.,

$$
\begin{aligned}
& \sum_{i=1}^{k} w_{i}(n)\left(z-\hat{x}_{i}\right)+\alpha \sum_{i=k+1}^{n} w_{i}(n)\left(z-\hat{x}_{i}\right) \\
& \leq \sum_{i=1}^{k} w_{i}(n)\left(z-\hat{y}_{i}\right)+\alpha \sum_{i=k+1}^{n} w_{i}(n)\left(z-\hat{y}_{i}\right),
\end{aligned}
$$

which, for sufficiently small $\alpha>0$, contradicts (6).

(b) $\Rightarrow\left(\right.$ a). Let $P_{w}$ be a poverty measure in $\mathcal{P}$. Let $x$ and $y$ be two income distributions in $X$ such that $n_{x}=n_{y}=n$. Assume that (b) holds. We have $\sum_{i=1}^{k} w_{i}(n) \hat{x}_{i} \geq \sum_{i=1}^{k} w_{i}(n) \hat{y}_{i}$ for each $k=1,2, \ldots, n$. This is equivalent to the condition that the vector $w_{x}=\left(w_{1}(n) \hat{x}_{1}, w_{2}(n) \hat{x}_{2}, \ldots, w_{n}(n) \hat{x}_{n}\right)$ is weakly supermajorized by the vector $w_{y}=\left(w_{1}(n) \hat{y}_{1}, w_{2}(n) \hat{y}_{2}, \ldots, w_{n}(n) \hat{y}_{n}\right)$ (Marshall, Olkin and Arnold, 2011, Definition A.2, p. 12). Let $P_{v}$ be an arbitrary poverty measure in $\mathcal{P}$ that is at least as distribution-sensitive as $P_{w}$. Then $P_{v}(x)=\sum_{i=1}^{q} \alpha_{i} w_{i}(n)\left(z-x_{i}\right) / z$ with $\alpha_{1} \geq \alpha_{2} \geq \cdots \geq \alpha_{n}>0$. Note that $\varphi: \mathbb{R}_{++}^{n} \rightarrow \mathbb{R}: t \mapsto \sum_{i=1}^{n} \alpha_{i} t_{i}$ is an increasing and Schur-concave function. Because $w_{x}$ is weakly supermajorized by $w_{y}$, we have $\varphi\left(w_{x}\right) \geq$ $\varphi\left(w_{y}\right)$ (Marshall, Olkin and Arnold, 2011, Theorem A.8, p. 87). It follows that $P_{v}(x) \leq P_{v}(y)$.

Proof of Proposition 5. $\left(\mathrm{a}^{\prime \prime}\right) \Rightarrow\left(\mathrm{b}^{\prime \prime}\right)$. Let $x$ and $y$ be two income distributions in $X$ such that $\hat{x} \neq \hat{y}$ and $n_{x}=n_{y}=n$. Assume that ( $\left.\mathrm{a}^{\prime \prime}\right)$ holds. Seeking a contradiction, assume that $\left(\mathrm{b}^{\prime \prime}\right)$ does not hold, i.e., $\hat{y}>_{\text {lex }} \hat{x}$. Let $k \leq n$ be such that $\hat{x}_{i}=\hat{y}_{i}$ for each $i<k$ and $\hat{y}_{k}>\hat{x}_{k}$. We have $\sum_{i=1}^{k} w_{i}(n)\left(z-\hat{x}_{i}\right)>\sum_{i=1}^{k} w_{i}(n)\left(z-\hat{y}_{i}\right)$ for each poverty measure $P_{w}$ in $\mathcal{P}$. Applying Proposition 4 , we obtain a contradiction.

$\left(\mathrm{b}^{\prime \prime}\right) \Rightarrow\left(\mathrm{a}^{\prime \prime}\right)$. Let $x$ and $y$ be two income distributions in $X$ such that $\hat{x} \neq \hat{y}$ and $n_{x}=n_{y}=n$. Assume that $\left(\mathrm{b}^{\prime \prime}\right)$ holds. Let $k \leq n$ be such that $\hat{x}_{i}=\hat{y}_{i}$ for each $i<k$ and $\hat{x}_{k}>\hat{y}_{k}$. Let $P_{w}$ be a rank-dependent poverty measure with a weight function in the form of equation (2) and

$$
f(t)= \begin{cases}\alpha t & \text { for each } t \leq \frac{n-k}{n}, \\ t-\frac{(1-\alpha)(n-k)}{n} & \text { for each } t \geq \frac{n-k}{n}\end{cases}
$$


where $0<\alpha<1$. Then

$$
w_{i}(n)= \begin{cases}\frac{1}{n} & \text { for each } i=1,2, \ldots, k, \\ \frac{\alpha}{n} & \text { for each } i=k+1, \ldots, n .\end{cases}
$$

Moreover, $P_{w}$ is in $\mathcal{P}$ since $f$ is strictly increasing, continuous and convex. Note that $\sum_{i=1}^{k} z-\hat{x}_{i}<\sum_{i=1}^{k} z-\hat{y}_{i}$. Hence, choosing $\alpha>0$ sufficiently small, we get

$$
\frac{1}{n} \sum_{i=1}^{k} z-\hat{x}_{i}+\frac{\alpha}{n} \sum_{i=k+1}^{\ell} z-\hat{x}_{i}<\frac{1}{n} \sum_{i=1}^{k} z-\hat{y}_{i}+\frac{\alpha}{n} \sum_{i=k+1}^{\ell} z-\hat{y}_{i}
$$

for each $\ell=k+1, k+2, \ldots, n$. Using Proposition 4, we obtain $\left(\mathrm{a}^{\prime \prime}\right)$. 